\title{
Size Matters: Better Staging of Lung Cancer, Better Outcomes!
}

\author{
Krasas A, Iliadis K
}

Lung cancer is the leading cause of cancer related deaths worldwide, and cancer is the second cause of death after cardiovascular diseases. In 2008, more than 1.6 million people were given a new diagnosis of lung cancer, comprising $13 \%$ of all cancer diagnoses, and 1.4 million died of lung cancer, which was $18 \%$ of all cancer deaths. The 5 -year survival rate for lung cancer in the United States is $15.6 \%$, and although there has been some improvement in survival during the past few decades, the advances that have been achieved in the case of other common malignancies have yet to be documented for lung cancer.

It is obvious that defining the anatomical extent of this very ferocious enemy is very important. The Tumor, Nodes, Metastasis (TNM) classification was first developed by Pierre Denoit in the 1950s to code the anatomical extent of tumors, since when, 7 revisions have been made concerning lung tumors. The classification system remains the most consistent prognosticator of the disease, but it does not explain the ultimate prognosis as this is multifactorial, and thus it does not dictate treatment.

In the $8^{\text {th }}$ Edition of TNM Classification, emphasis has been placed on the tumor size, and the cut-off points have been further proliferated in such a way that size will now be a descriptor in all $\mathrm{T}$ categories. The division of the category $\mathrm{T} 1$ into $\mathrm{T} 1 \mathrm{a}, \mathrm{T} 1 \mathrm{~b}$ and $\mathrm{T} 1 \mathrm{c}$ on the basis of the new size cut points of $1 \mathrm{~cm}$ and $2 \mathrm{~cm}$ has resulted in these new case categories, reflecting the statistically different prognosis of these categories. These new cut points and the stage groupings should be used in any trials of novel therapies, such as sublobar resection, segmentectomy, and non-surgical treatment options. Regarding segmentectomy, it appears to offer a good oncological result for small tumors (i.e., $<2 \mathrm{~cm}$ ), and with mediastinal lymph node dissection offers correct pathological staging. Segmentectomy is, for this reason, an excellent alternative for patients with compromised respiratory function, and in the future will be introduced increasingly into the surgical armamentarium. The new cut-off points should not in themselves be taken as a constraint on the use of structured surveillance in studies of CT screening, because the proportion of tumor detected by screening was unknown in the data set on which the $8^{\text {th }}$ edition was based.

The overall survival by clinical stage, according to the $8^{\text {th }}$ edition, showed significant improvement at 2 years and 5 years, as it is nicely described in this issue's article by Nikolouzos S., et al.

\section{Reference}

1. Nikolouzos S., Zacharia G., Baltayiannis N., et al. Comments on the Eighth Edition of the TNM Classification for Lung Cancer. Radical Changes and Limitations: Does Size Matter? Hellenic J Surg 2017;89:141-8.
Krasas A MD, Iliadis K MD

Department of Thoracic Surgery, "Hygeia" Hospital, Athens, Greece

Corresponding author: Krasas Athanasios

3 Thessalonikis St,- Halandri 15234, Athens, Greece

Tel.: 6932454341

e-mail: athanasekrassas@hotmail.fr 